\title{
A VERY SINGULAR SOLUTION OF A QUASILINEAR DEGENERATE DIFFUSION EQUATION WITH ABSORPTION
}

\section{A. PELETIER AND JUNYU WANG}

\begin{abstract}
The object of this paper is to study the existence of a nonnegative solution of the Cauchy problem

$$
u_{t}=\operatorname{div}\left(|\nabla u|^{p-2} \nabla u\right)-u^{q}, \quad u(x, 0)=0 \quad \text { if } x \neq 0,
$$

which is more singular at $(0,0)$ than the fundamental solution of the corresponding equation without the absorption term.
\end{abstract}

1. Introduction. In a recent paper [1] Brezis, Peletier and Terman found a very singular solution of the heat equation with absorption

$$
u_{t}=\Delta u-u^{q} \quad \text { in } S=\mathbf{R}^{N} \times(0, \infty)
$$

when $1<q<1+(2 / N)$ and $N \geq 1$. By this was meant a solution $W(x, t)$ with the properties

(i) $W>0$ in $S$;

(ii) $W$ is smooth in $\bar{S}$, except at $(0,0)$;

(iii) $W(x, 0)=0$ for all $x \in \mathbf{R}^{N}$, except at $x=0$;

(iv) $W$ is more singular at the origin than the fundamental solution $E$ of the heat equation, specifically

$$
\int_{\mathbf{R}^{n}} W(x, t) d x \rightarrow \infty \quad \text { as } t \downarrow 0 .
$$

This very singular solution turned out to play an important role in the behavior of more general solutions of $(1.1)$ as $t \rightarrow 0[\mathbf{7}]$ and as $t \rightarrow \infty[2,6]$.

A corresponding very singular solution for the Porous Media Equation with absorption

$$
u_{t}=\Delta\left(u^{m}\right)-u^{q}
$$

in which $m>1$, was found to exist by Peletier and Terman [8] when $m<q<$ $m+(2 / N)$.

Motivated by these results we shall show in this paper that the quasilinear degenerate diffusion equation - involving the $p$-laplacian-with absorption

$$
u_{t}=\operatorname{div}\left(|\nabla u|^{p-2} \nabla u\right)-u^{q} \quad \text { in } S
$$

also has a very singular solution under suitable restrictions on $p$ and $q$. Here we shall assume throughout that $p>2$.

Received by the editors October 6, 1986 and, in revised form, April 27, 1987.

1980 Mathematics Subject Classification (1985 Revision). Primary 35K65; Secondary 35K15, $35 \mathrm{~K} 55$.

(C)1988 American Mathematical Society $0002-9947 / 88 \$ 1.00+\$ .25$ per page 
By a very singular solution of equation (1.3) we mean a function $W(x, t)$ which satisfies (1.3) in some sense, and possesses the properties

$$
\begin{aligned}
& W \geq 0 \text { in } \bar{S} \backslash\{(0,0)\} \\
& W \text { is continuous in } \bar{S} \backslash\{(0,0)\} ; \\
& W(x, 0)=0 \text { for all } x \in \mathbf{R}^{N} \backslash\{0\} ; \\
& W \text { is more singular than } E \text { at }\{(0,0)\} .
\end{aligned}
$$

In this definition $E$ denotes the singular solution of equation (1.3) without absorption

with $p>2$. It is given by

$$
u_{t}=\operatorname{div}\left(|\nabla u|^{p-2} \nabla u\right)
$$

where

$$
E(x, t)=t^{-1 / \mu} \psi(\xi), \quad \xi=|x| t^{-1 / N \mu}
$$

$$
\mu=p-2+(p / N)
$$

and $\psi$ is given by

$$
\psi(\xi)=c(p, N)\left\{\left[\xi_{0}^{p /(p-1)}-\xi^{p /(p-1)}\right]_{+}\right\}^{(p-1) /(p-2)} .
$$

Here $\xi_{0}$ is an arbitrary positive constant,

$$
c(p, N)=\left(\frac{p-2}{p}\right)^{(p-1) /(p-2)}(N \mu)^{-1 /(p-2)}
$$

and $[z]_{+}=\max \{0, z\}$.

As in [1 and 8] we look for a very singular solution of the form

$$
W(x, t)=t^{-1 /(q-1)} f(\eta), \quad \eta=|x| t^{-1 / \beta} .
$$

Such a function $W$ will satisfy (1.3)-(1.6) if

$$
\beta=p(q-1) /(q+1-p)
$$

and if $f$ is a solution of the problem

$$
\text { (I) }\left\{\begin{array}{l}
\left(\left|f^{\prime}\right|^{p-2} f^{\prime}\right)^{\prime}+\frac{N-1}{\eta}\left|f^{\prime}\right|^{p-2} f^{\prime}+\frac{1}{\beta} \eta f^{\prime}+\frac{1}{q-1} f-f^{q}=0 \quad \text { in }(0, \infty), \\
f(\eta) \geq 0 \quad \text { on }[0, \infty), \\
f^{\prime}(0)=0, \quad \lim _{\eta \rightarrow \infty} \eta^{p /(q+1-p)} f(\eta)=0 .
\end{array}\right.
$$

We shall show that such a solution indeed exists for appropriate values of $p$ and $q$.

THEOREM. Suppose that $N \geq 1, p>2$ and

$$
p-1<q<p-1+p / N \text {. }
$$

Then there exists a nontrivial solution $f$ of Problem (I); $f$ has compact support.

The conditions on $p$ and $q$ in this theorem ensure that the singularity of $W(x, t)$ at $(0,0)$ is stronger than that of $E(x, t)$. For instance

$$
E(0, t)=\psi(0) t^{-1 / \mu} \quad \text { and } W(0, t)=f(0) t^{-1 /(q-1)},
$$

and, by (1.8) and (1.13), $q-1<p-2+(p / N)=\mu$. 
The solution $f(\eta)$ we obtain is of the form

$$
f(\eta) \begin{cases}>0 & \text { for } 0 \leq \eta<\eta_{0} \\ =0 & \text { for } \eta_{0} \leq \eta<\infty\end{cases}
$$

where $\eta_{0}$ is some positive number. At the point $\eta_{0}$, we find that

$$
\lim _{\eta \uparrow \eta_{0}} \frac{\left|f^{\prime}(\eta)\right|^{p-2} f^{\prime}(\eta)}{f(\eta)}=-\frac{\eta_{0}}{\beta} .
$$

Observe that the lower bound for $q$ in (1.13) ensures that $\beta>0$; the upper bound will be needed to ensure the behavior of $f(\eta)$ as $\eta \rightarrow \infty$, required by (1.12).

It is interesting to note that for the corresponding elliptic equation

$$
-\operatorname{div}\left(|\nabla u|^{p-2} \nabla u\right)+u^{q}=0, \quad u \geq 0
$$

there exists a very singular solution if

$$
p-1<q<N \frac{p-1}{N-p} \quad \text { if } 1<p<N
$$

or

$$
p-1<q \text { if } p=N
$$

It is given explicitly by

$$
u(x)=\gamma_{N, p, q}|x|^{-p /(q+1-p)}
$$

where

$$
\gamma_{N, p, q}=\left[\left(\frac{p}{q+1-p}\right)^{p-1}\left(\frac{p q}{q+1-p}-N\right)\right]^{1 /(q+1-p)} .
$$

For further details we refer to Friedman and Veron who give in [3 and 4] a complete classification of the isolated singularities of equation (1.15).

In this paper we have imposed the condition $p>2$, leaving the range $1<p<2$ unexplored. We intend to return to this range in a forthcoming paper.

The organization of this paper is the following. In $\S 2$ we place Problem (I) in the context of a class of problems, and derive a few properties of its solutions. In particular we shall prove that a solution is a decreasing function whenever it is positive. In $\S 3$, we formulate equation (1.10) as a system of three first order differential equations by introducing appropriate variables, dependent as well as independent. In this setting the desired solution corresponds to an orbit connecting two given curves in the three-dimensional solution space. In $\S 4$ we establish the existence of such an orbit by means of a shooting argument, not unlike that used in [8].

2. Preliminaries. We shall consider the more general boundary value problem

$$
\text { (II) }\left\{\begin{array}{l}
\left(\left|u^{\prime}\right|^{p-2} u^{\prime}\right)^{\prime}+\frac{N-1}{x}\left|u^{\prime}\right|^{p-2} u^{\prime}+\frac{x}{\beta} u^{\prime}+f(u)=0, \quad x>0 \\
u(x) \geq 0 \quad(\not \equiv 0), \quad x \geq 0 \\
u^{\prime}(0)=0, \quad \lim _{x \rightarrow \infty} u(x)=0
\end{array}\right.
$$


in which $p>2, \beta>0, N \geq 1$ and

$$
f(u)=(\alpha / \beta) u-u^{q},
$$

where $\alpha>0$ and $q>1$. Clearly we obtain Problem (I) if we set

$$
\alpha=\frac{p}{q-p+1}, \quad \beta=\frac{p(q-1)}{q-p+1}
$$

and require in addition that

$$
u(x)=o\left(x^{-\alpha}\right) \quad \text { as } x \rightarrow \infty .
$$

By a solution of Problem (II) we shall mean a function $u \in C^{1}([0, \infty))$ such that $\left|u^{\prime}\right|^{p-2} u^{\prime} \in C^{1}(0, \infty)$ which satisfies (2.1)-(2.3). In fact, $\left|u^{\prime}\right|^{p-2} u^{\prime} \in C^{1}([0, \infty))$ and it is readily shown that

$$
\lim _{x \downarrow 0} \frac{\left|u^{\prime}(x)\right|^{p-2} u^{\prime}(x)}{x}=\left(\left|u^{\prime}\right|^{p-2} u^{\prime}\right)^{\prime}(0)=-\frac{1}{N} f(u(0)) .
$$

The main result of this paper is the following existence theorem.

THEOREM 1. Suppose $N \geq 1, p>2, \beta>0$ and

$$
\alpha>N, \quad q>1 \text {. }
$$

Then Problem (II) has a solution $u$ with the property $\lim _{x \rightarrow \infty} x^{\alpha} u(x)=0$.

We begin, in this section, by deriving a few properties of solutions of Problem (II).

Lemma 1. Suppose $u(x)$ is a solution of Problem (II) in which $\alpha \geq N$. Then

(i) $u(x) \leq A$ for all $x \geq 0 ; A=(\alpha / \beta)^{1 /(q-1)}$;

(ii) $u(x)$ is nonincreasing on $[0, \infty)$;

(iii) $u^{\prime}(x)<0$ at points $x>0$ where $u(x)>0$.

PROOF. (i) Suppose to the contrary that

$$
u(\bar{x})=\sup \{u(x): x>0\}>A .
$$

Then at $x=\bar{x}: u>A, u^{\prime}=0,\left(\left|u^{\prime}\right|^{p-2} u^{\prime}\right)^{\prime} \leq 0$. If $\bar{x}>0$, we deduce, however, from the differential equation that

$$
\left(\left|u^{\prime}\right|^{p-2} u^{\prime}\right)^{\prime}(\bar{x})=-f(u(\bar{x}))>0
$$

i.e. a contradiction. If, on the other hand, $\bar{x}=0$, we obtain a contradiction to (2.8) from (2.7). Therefore (2.8) must be false, and $u \leq A$ on $[0, \infty)$.

(ii) Suppose that for some $x_{0}>0, u^{\prime}\left(x_{0}\right)>0$. Then in view of (2.7) there exists a point $\bar{x} \in\left(0, x_{0}\right)$ such that at $\bar{x}$

$$
0 \leq u<A, \quad u^{\prime}=0, \quad\left(|u|^{p-2} u^{\prime}\right)^{\prime} \geq 0 .
$$

We shall show in Lemma 2 that $u(\bar{x})>0$. This means, according to equation (2.1) that $\left(\left|u^{\prime}\right|^{p-2} u^{\prime}\right)^{\prime}<0$ at $x=\bar{x}$, contradicting (2.9). Thus $u^{\prime}(x) \leq 0$ for all $x \geq 0$.

(iii) It follows from the proof of part (ii) that $u^{\prime}(x)<0$ at every $x>0$, where $0<u(x)<A$. Thus it remains to exclude the case that for some $a>0$,

$$
u(x) \begin{cases}=A, & 0 \leq x \leq a, \\ <A, & a<x<\infty .\end{cases}
$$


We assert that there exists a $\delta>0$ such that $u^{\prime}(x)<0$ for $a<x<a+\delta$. For, if no such $\delta$ can be found, there exists a sequence $\left\{x_{n}\right\}, x_{n} \downarrow a$, such that $u^{\prime}\left(x_{n}\right)=0$ and $0<u\left(x_{n}\right)<A$. By equation (2.1) the points $\left\{x_{n}\right\}$ can only be strict maxima. Since by the continuity of $u^{\prime}$, there will be minima between the points $\left\{x_{n}\right\}$, where $u^{\prime}=0$, equation (2.1) yields a contradiction.

If we multiply equation $(2.1)$ by $x^{N-1}$, we can write it as

$$
\left(x^{N-1}\left|u^{\prime}\right|^{p-2} u^{\prime}\right)^{\prime}+\frac{1}{\beta} x^{N} u^{\prime}+x^{N-1} f(u)=0 .
$$

Integration over $(a, x)$, where $a<x<a+\delta$, now yields, after some elementary manipulations:

$$
\begin{aligned}
& x^{N-1}\left|u^{\prime}\right|^{p-1}+\frac{x^{N}}{\beta}[A-u(x)] \\
& \quad=\frac{N}{\beta} \int_{a}^{x} s^{N-1}[A-u(s)] d s+\int_{a}^{x} s^{N-1} f(u(s)) d s .
\end{aligned}
$$

Because $u$ is decreasing on $(a, a+\delta)$

$$
\int_{a}^{x} s^{N-1}[A-u(s)] d s \leq \frac{1}{N}\left(x^{N}-a^{N}\right)[A-u(x)]
$$

and, remembering the definition (2.4) of $f$ :

$$
\begin{aligned}
\int_{a}^{x} s^{N-1} f(u(s)) d s & \leq q A^{q-1} \int_{a}^{x} s^{N-1}[A-u(s)] d s \\
& \leq \frac{q A^{q-1}}{N}\left(x^{N}-a^{N}\right)[A-u(x)] .
\end{aligned}
$$

Hence, if we substitute (2.12) and (2.13) into (2.11), and divide by $x^{N}[A-u(x)]$, we obtain

$$
\frac{1}{\beta} \leq\left(\frac{1}{\beta}+\frac{q}{N} A^{q-1}\right)\left\{1-\left(\frac{a}{x}\right)^{N}\right\}
$$

If we now let $x$ tend to $a$, we arrive at the contradiction $1 / \beta \leq 0$.

Thus, we have excluded the possibility that $u=A$ on some interval $[0, a], a>0$. This completes the proof of the lemma.

In the following lemma we discuss the behavior of a nonnegative solution of (2.1) near a zero.

LEMMA 2. Let $u$ be a nonnegative solution of equation (2.1) such that at some point $x_{0} \geq 0, u\left(x_{0}\right)=u^{\prime}\left(x_{0}\right)=0$. Suppose $\alpha \geq N$. Then

(i) $u(x) \equiv 0$ for all $x \geq x_{0}$;

(ii) if $x_{0}>0$ and $u>0$ in a left neighborhood of $x_{0}$, then

$$
\lim _{x \uparrow x_{0}} u^{-1}\left|u^{\prime}\right|^{p-1}=x_{0} / \beta .
$$

Proof. (i) If not, we may assume that $u(x)>0$ and $u^{\prime}(x)>0$ for $x \in$ $\left(x_{0}, x_{0}+\varepsilon\right)$, where $\varepsilon$ is some sufficiently small positive number.

We multiply the equation by $x^{N-1}$ and integrate over $\left(x_{0}, x\right)$, where $x_{0}<x<$ $x_{0}+\varepsilon$. This yields

$$
x^{N-1}\left|u^{\prime}\right|^{p-1}+\frac{x^{N}}{\beta} u+\frac{\alpha-N}{\beta} \int_{x_{0}}^{x} s^{N-1} u(s) d s=\int_{x_{0}}^{x} s^{N-1} u^{q}(s) d s .
$$


Hence, because $\alpha \geq N$,

$$
\frac{x^{N}}{\beta} u(x) \leq \int_{x_{0}}^{x} s^{N-1} u^{q}(s) d s \leq \frac{1}{N}\left(x^{N}-x_{0}^{N}\right) u^{q}(x)
$$

or

$$
\frac{1}{\beta} \leq \frac{1}{N} u^{q-1}(x)\left\{1-\left(\frac{x_{0}}{x}\right)^{N}\right\} .
$$

If we now let $x$ tend to $x_{0}$, we arrive at a contradiction.

(ii) Choose $\varepsilon>0$ so small that $u>0$ and $u^{\prime}<0$ in $\left(x_{0}-\varepsilon, x_{0}\right)$. Then, as in part (i) we obtain

$$
x^{N-1}\left|u^{\prime}\right|^{p-1}+\frac{\alpha-N}{\beta} \int_{x}^{x_{0}} s^{N-1} u(s) d s=\frac{x^{N}}{\beta} u+\int_{x}^{x_{0}} s^{N-1} u^{q}(s) d s
$$

when $x \in\left(x_{0}-\varepsilon, x_{0}\right)$. Hence, because $\alpha \geq N$,

$$
\frac{\left|u^{\prime}(x)\right|^{p-1}}{u(x)} \leq \frac{x}{\beta}+\frac{\int_{x}^{x_{0}} s^{N-1} u^{q}(s) d s}{x^{N-1} u(x)}
$$

and

$$
\frac{\left|u^{\prime}(x)\right|^{p-1}}{u(x)}>\frac{x}{\beta}-\frac{\alpha-N}{\beta} \frac{\int_{x}^{x_{0}} s^{N-1} u(s) d s}{x^{N-1} u(x)} .
$$

If we let $x \uparrow x_{0}$ in these inequalities, we obtain the required limit.

REMARK. The limit in part (ii) of Lemma 2 is equivalent to

$$
\lim _{x \uparrow x_{0}}\left|\frac{p-1}{p-2} v^{\prime}\right|^{p-1}=\frac{x_{0}}{\beta},
$$

where $v=u^{(p-2) /(p-1)}$.

3. The system. The limit (2.14) suggests we introduce the new dependent variables

$$
v=u^{(p-2) /(p-1)} \quad \text { and } \quad w=-\frac{p-1}{p-2} v^{\prime} .
$$

Equation (2.1) can then be written as the first order system

$$
\text { (III) }\left\{\begin{array}{l}
v^{\prime}=-\frac{p-2}{p-1} w \\
v\left(w^{p-1}\right)^{\prime}=w^{p}-\frac{N-1}{x} v w^{p-1}-\frac{x}{\beta} w+\frac{\alpha}{\beta} w-v^{1+\mu}
\end{array}\right.
$$

where $\mu=(q-1)(p-1) /(p-2)$, because $w \geq 0$ by Lemma 1 .

To simplify the left side of (3.2), we also introduce a new independent variable $t$ through the symbolic equation

$$
\frac{d}{d t}=(p-1) v w^{p-2} \frac{d}{d x} .
$$

This yields the system

$$
(\mathrm{IV})\left\{\begin{array}{l}
v^{\prime}=-(p-2) v w^{p-1} \\
w^{\prime}=w^{p}-\frac{N-1}{x} v w^{p-1}-\frac{x}{\beta} w+g(v) \\
x^{\prime}=(p-1) v w^{p-2}
\end{array}\right.
$$


where primes now denote differentiation with respect to $t$ and

$$
g(v)=(\alpha / \beta) v-v^{1+\mu} .
$$

By Lemmas 1 and 2 it suffices to find a solution $(v(t), w(t), x(t))$ defined for $t>0$ with the properties

$$
0<v(0) \leq B, \quad w(0)=0, \quad x(0)=0
$$

and

$$
\lim _{t \rightarrow \infty} v(t)=0, \quad \lim _{t \rightarrow \infty} x(t)=\lim _{t \rightarrow \infty} \beta w^{p-1}(t)<\infty
$$

where

$$
B=A^{(p-2) /(p-1)}=(\alpha / \beta)^{1 / \mu} .
$$

The set of critical points of (IV) in the half space $x>0$ consists of the two half lines

$$
\begin{aligned}
& L_{1}=\{(v, w, x): v=0, w=0, x>0\} \\
& L_{2}=\{(v, w, x): v=B, w=0, x>0\}
\end{aligned}
$$

and the curve

$$
C=\left\{(v, w, x): v=0, w^{p-1}=x / \beta, x>0\right\}
$$

(See Figure 1.)

On this curve $C$ we define for every $\gamma>0$ the point

$$
P_{\gamma}=\left(0,(\gamma / \beta)^{1 /(p-1)}, \gamma\right)
$$

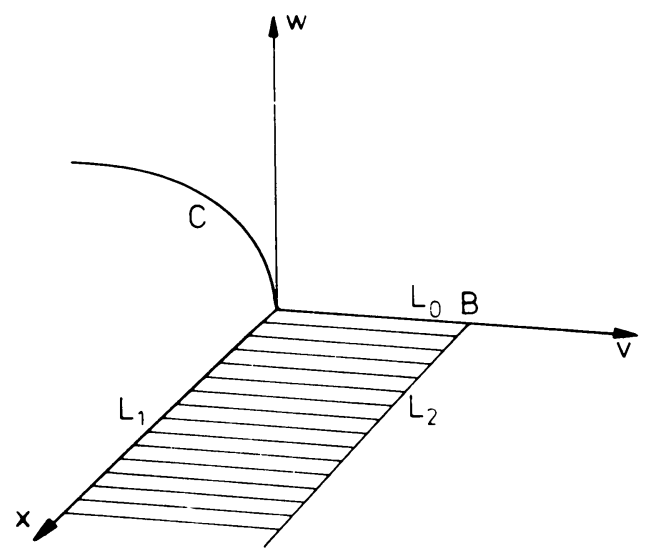

Figure 1. The sets $L_{1}, L_{2}$ and $C$ 
Near $P_{\gamma}$, the flow of (IV) is described by the linear system obtained by linearizing (IV) at $P_{\gamma}$. The coefficient matrix $M$ of this system is given by

$$
M=\left(\begin{array}{lcc}
-(p-2) \frac{\gamma}{\beta} & 0 & 0 \\
\frac{\alpha-N+1}{\beta} & (p-1) \frac{\gamma}{\beta} & -\frac{1}{\beta}\left(\frac{\gamma}{\beta}\right)^{1 /(p-1)} \\
(p-1)\left(\frac{\gamma}{\beta}\right)^{(p-2) /(p-1)} & 0 & 0
\end{array}\right)
$$

Its eigenvalues are

$$
\lambda_{1}=-(p-2) \frac{\gamma}{\beta}, \quad \lambda_{2}=(p-1) \frac{\gamma}{\beta}, \quad \lambda_{3}=0
$$

and their corresponding eigenvectors:

$$
\begin{aligned}
& e_{1}=\left((p-2) \frac{\gamma}{\beta},-\frac{(\alpha-N)(p-2)+2 p-3}{(2 p-3) \beta},-(p-1)\left(\frac{\gamma}{\beta}\right)^{(p-2) /(p-1)}\right), \\
& e_{2}=(0,1,0), \\
& e_{3}=\left(0,(\gamma / \beta)^{1 /(p-1)},(p-1) \gamma\right) .
\end{aligned}
$$

In view of (3.4) we look for an orbit which enters a point $P_{\gamma}$ on $C$. For such orbits we have the following result [5, p. 127].

LEMMA 3. For each $\gamma>0$ there exists a unique (up to translation) nonconstant solution

$$
U(t, \gamma)=(v(t, \gamma), w(t, \gamma), x(t, \gamma))
$$

of (IV) such that $\lim _{t \rightarrow \infty} U(t, \gamma)=P_{\gamma}$. In addition, as $t \rightarrow \infty, U(t, \gamma)$ enters $P_{\gamma}$ along the eigenvector $e_{1}$ at $P_{\gamma}$.

Because $\lambda_{1}$ and $e_{1}$ depend continuously on $\gamma$, we may choose the translation so that $U(0, \gamma)$ is a continuous function. Thus, from classical results on the continuous dependence of solutions of ordinary differential equations on the initial value and a parameter in the equation, we may deduce the following lemma.

LEMMA 4. Let $\gamma^{*}>0$. For given constants $\varepsilon, T>0$ there exists a constant $\delta>0$ such that if $\left|\gamma-\gamma^{*}\right|<\delta$, then

$$
\left|U(t, \gamma)-U\left(t, \gamma^{*}\right)\right| \leq \varepsilon \quad \text { for all } t \geq T .
$$

Here $|\cdot|$ denotes the usual norm in $\mathbf{R}^{3}$.

To prove the existence of a solution $u$ of Problem (II) which converges to zero at infinity sufficiently fast, we use a shooting argument, shooting backward from $P_{\gamma}$ for different values of $\gamma>0$. The idea of the proof will then be to show that there exists a $\gamma_{0}>0$ and a time $T_{0} \in(-\infty, \infty)$ such that

$$
\lim _{t \downarrow T_{0}} U\left(t, \gamma_{0}\right) \in L_{0} \stackrel{\text { def }}{=}\{(v, w, x): v>0, w=0, x=0\} .
$$

The function $U\left(t, \gamma_{0}\right)$ is then readily found to correspond to a solution of Problem (II). 


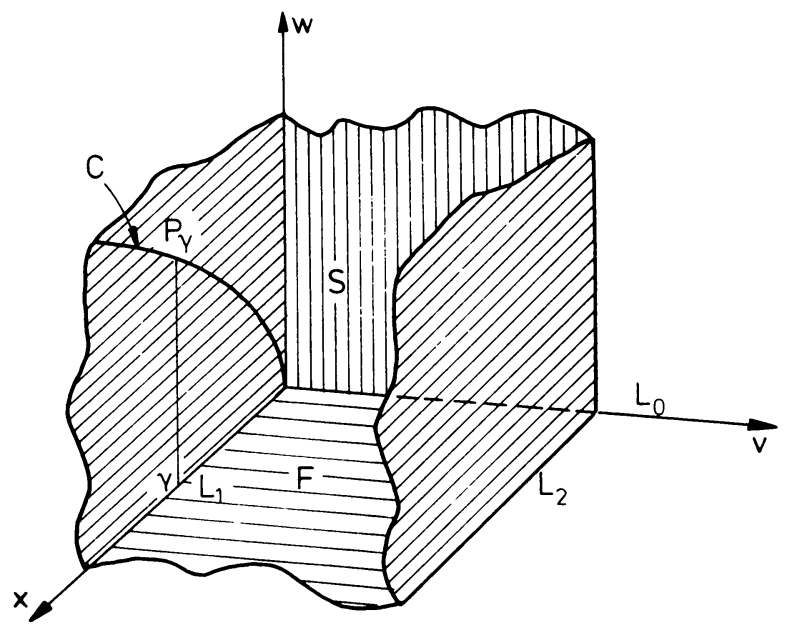

Figure 2. The sets $S$ and $F$

4. The existence proof. We shall need the sets

$$
\begin{aligned}
& S=\{(v, w, x): 0<v<B, w>0, x>0\}, \\
& F=\{(v, w, x): 0<v<B, w=0, x>0\},
\end{aligned}
$$

where $B$ was defined in (3.5). (See Figure 2.)

Because the vector $e_{1}$ points from $P_{\gamma}$ into $S$, the orbit $U(t, \gamma)$, when it emerges from $P_{\gamma}$ as time runs backward, will enter $S$. We shall denote the time when it leaves $S$ again-time still running backward-by $T_{\gamma}$ :

$$
T_{\gamma}=\sup \{t: U(t, \gamma) \notin S\} \text {. }
$$

If it does not leave $S$, i.e. $U(t, \gamma) \in S$ for all $t \in\left(T_{\min }, \infty\right)$, where $\left(T_{\min }, \infty\right)$ is its maximal interval of existence, we set $T_{\gamma}=T_{\min }$.

We shall denote the set of values of $\gamma \in \mathbf{R}^{+}$for which $U(t, \gamma)$ leaves $S$ through the bottom by $G$ :

$$
G=\left\{\gamma>0: T_{\gamma}>-\infty \text { and } U\left(T_{\gamma}, \gamma\right) \in F\right\} .
$$

LEMMA 5. Suppose $\alpha>N$. Then there exists a number $\gamma_{1}>0$ such that if $\gamma \in\left(0, \gamma_{1}\right)$, then $\gamma \in G$.

Proof. Choose

$$
\gamma_{1}=\min \left\{\left(\frac{\alpha-N}{\beta}\right)^{1 / \mu}, \beta\left(\frac{p-1}{p-2}\right)^{p-1}\right\}
$$

and define the set

$$
S_{1}=\left\{(v, w, x): 0<v<\gamma_{1}, 0<x<\gamma_{1}-v, 0<w<(x / \beta)^{1 /(p-1)}\right\} .
$$

(See Figure 3.) 


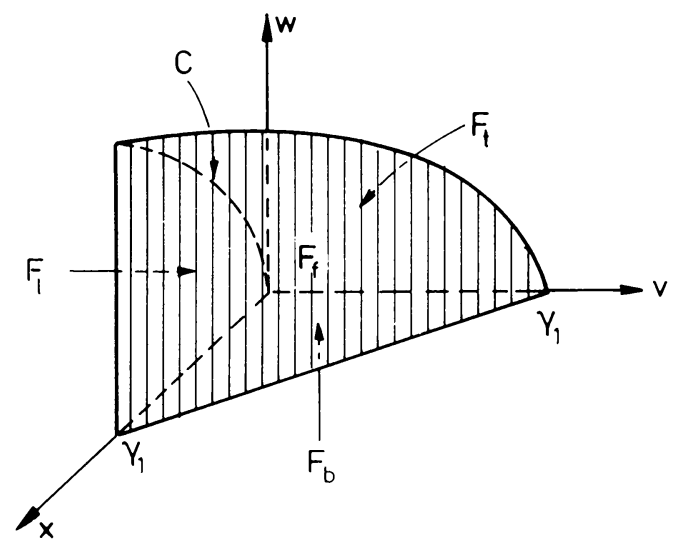

Figure 3 . The set $S_{1}$

We compute where on the boundary $\partial S_{1}$ of $S_{1}, U(t, \gamma)$ may enter or leave $S_{1}$.

On the front $F_{f}$ of $S_{1}$,

$$
F_{f}=\left\{(v, w, x): 0<v<\gamma_{1}, x=\gamma_{1}-v, 0<w<(x / \beta)^{1 /(p-1)}\right\}
$$

the outward pointing normal vector is given by $n_{f}=(1,0,1)$ (not normalized). Let $X$ denote the vector field determined by (IV). Then

$$
\begin{aligned}
n_{f} \cdot X & =v w^{p-2}\{(p-1)-(p-2) w\} \\
& >v w^{p-2}\left\{(p-1)-(p-2)\left(\gamma_{1} / \beta\right)^{1 /(p-1)}\right\}>0
\end{aligned}
$$

in view of the definition of $\gamma_{1}$. Thus, $F_{f}$ is part of the exit set of $S_{1}$.

On the top $F_{t}$ of $S_{1}$,

$$
F_{t}=\left\{(v, w, x): 0<v<\gamma_{1}, 0<x<\gamma_{1}-v, x=\beta w^{p-1}\right\}
$$

the outward pointing normal $n_{t}$ is given by

$$
n_{t}=\left(0,(p-1) \beta w^{p-2},-1\right)
$$

and

$$
\begin{aligned}
n_{t} \cdot X & =(p-1) \beta w^{p-2}\left\{g(v)-\frac{N-1}{x} v w^{p-1}\right\}-(p-1) v w^{p-2} \\
& =(p-1) v w^{p-2}\left\{(\alpha-N)-\beta v^{\mu}\right\}>0,
\end{aligned}
$$

where we have used the definition of $\gamma_{1}$ again. Thus $F_{t}$ is also part of the exit set of $S_{1}$.

On the left-hand side $F_{l}$ of $S_{1}$,

$$
F_{l}=\left\{(v, w, x): v=0,0<x<\gamma_{1}, 0<w<(x / \beta)^{1 /(p-1)}\right\},
$$

we have $v^{\prime}=0, x^{\prime}=0$ and

$$
w^{\prime}=w\left\{w^{p-1}-(x / \beta)\right\}<0 .
$$

Therefore, this side is invariant, and orbits in $F_{l}$ converge to $L_{1}$.

Finally, on the bottom $F_{b}$ of $S_{1}$ :

$$
F_{b}=\left\{(v, w, x): 0<v<\gamma_{1}, w=0,0<x<\gamma_{1}-v\right\}
$$


we have $w^{\prime}=g(v)>0$ because $\gamma_{1}<B$. Hence $F_{b}$ is part of the entrance set of $S_{1}$.

Thus, we have shown that the entrance set of $S_{1}$ consists of only $F_{b}$, and therefore that if a trajectory leaves $S_{1}$ when time runs backward, it must do so through $F_{b}$.

Because $F_{b} \subset F$, it remains to prove that (i) the trajectory $U(t, \gamma)$ enters $S_{1}$ from $P_{\gamma} \in C$ when $t$ decreases from $+\infty$, and (ii) that it must leave $S_{1}$ again at some $t_{0}>-\infty$. Now, according to Lemma $3, U(t, \gamma)$ enters $P_{\gamma}$ along the vector $-e_{1}$ at $P_{\gamma}$. Thus as regards (i) it suffices to prove that the vector

$$
e_{1}=\left((p-2) \frac{\gamma}{\beta},-\frac{(\alpha-N)(p-2)+2 p-3}{(2 p-3) \beta},-(p-1)\left(\frac{\gamma}{\beta}\right)^{(p-2) /(p-1)}\right)
$$

points from $P_{\gamma}$ into $S_{1}$.

The point $P_{\gamma}$ lies on the curve where the left-hand side $F_{l}$ and the top $F_{t}$ of $S_{1}$ intersect. The outward pointing normals on $F_{l}$ and $F_{t}$ are

$$
n_{l}=(-1,0,0), \quad n_{t}=\left(0,(p-1) \beta(\gamma / \beta)^{(p-2) /(p-1)},-1\right) .
$$

Therefore

$$
e_{1} \cdot n_{l}=-(p-2) \gamma / \beta<0
$$

and

$$
e_{1} \cdot n_{t}=-\frac{(p-1)(p-2)}{2 p-3}(\alpha-N)\left(\frac{\gamma}{\beta}\right)^{(p-2) /(p-1)}<0
$$

because $\alpha>N$, whence indeed, $e_{1}$ points into $S_{1}$.

To prove (ii), suppose to the contrary that $U(t, \gamma) \in S_{1}$ for all $t \in \mathbf{R}$. Then, by (IV),

$$
v(t) \nearrow \bar{v}, \quad x(t) \searrow \bar{x} \quad \text { as } t \rightarrow-\infty,
$$

where $0<\bar{v} \leq \gamma_{1}$ and $0 \leq \bar{x}<\gamma_{1}$. In addition

$$
\liminf _{t \rightarrow-\infty} w(t)=0,
$$

since otherwise $x(t) \rightarrow-\infty$ as $t \rightarrow-\infty$, and $U(t, \gamma)$ would leave $S_{1}$ as $t$ decreases. Also, because $w^{p-1}(t)<x(t) / \beta$ when $U(t, \gamma) \in S_{1}$,

$$
w^{\prime} \geq w^{p}-\frac{x}{\beta} w+\frac{\alpha-N+1}{\beta} v-v^{1+\mu}>w^{p}-\frac{x}{\beta} w+\frac{1}{\beta} v
$$

because $\alpha>N$ and $v<\gamma_{1} \leq\{(\alpha-N) / \beta\}^{1 / \mu}$. Thus, in view of (4.1),

$$
w^{\prime}>w^{p}-\frac{\gamma_{1}}{\beta} w+\frac{1}{2 \beta} \bar{v}
$$

for $-t$ large enough.

It follows from (4.3) that there exists a number $w_{1}>0$ such that if for some time $t_{1} \in \mathbf{R}, w\left(t_{1}\right)<w_{1}$, then there exists a time $t_{2}<t_{1}$ such that $w\left(t_{2}\right)=0$ and $w^{\prime}\left(t_{2}\right)>0$. Thus, in that case, $U(t, \gamma)$ would leave $S_{1}$ at $t=t_{2}$ in backward time. Since, by assumption, this cannot happen, we must conclude that $w(t) \geq w_{1}$ for all $-t$ sufficiently large. This contradicts (4.2), and completes the proof of (ii). 


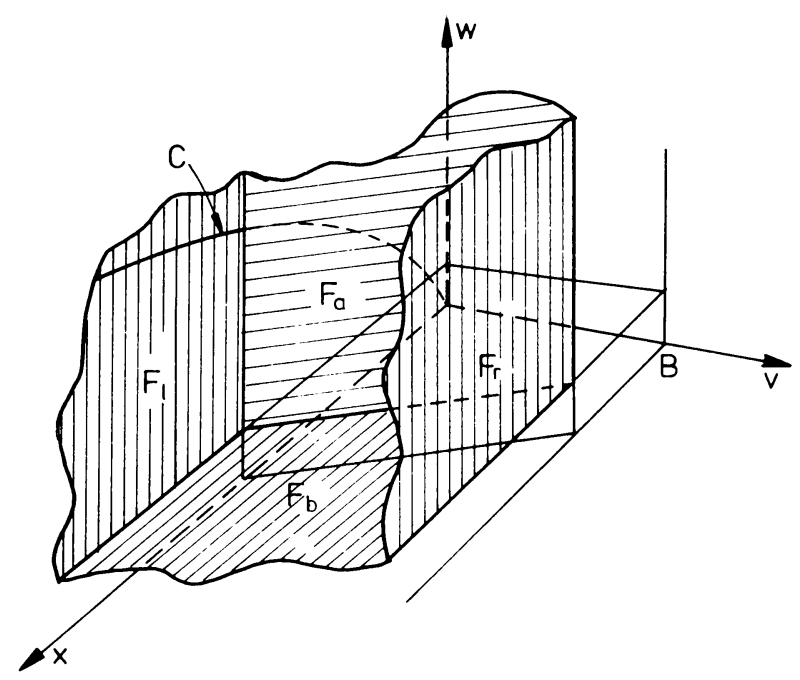

FIGURE 4 . The set $S_{2}$

LEMMA 6. There exists a number $\gamma_{2}>0$ such that if $\gamma>\gamma_{2}$, then $\gamma \notin G$.

ProOF. Set

$$
M=\max \{g(v): 0 \leq v \leq B\}, \quad \gamma_{2}=\beta(1+M)+\frac{p-1}{p-2} B
$$

and define the set $S_{2}$ (see Figure 4).

$$
S_{2}=\left\{(v, w, x): 0<v<B, w>1, x>\gamma_{2}-\frac{p-1}{p-2} v\right\} .
$$

As in the proof of Lemma 5, we determine the entrance set and the exit set of $S_{2}$.

On the bottom $F_{b}$ of $S_{2}$ :

$$
F_{b}=\left\{(v, w, x): 0<v<B, w=1, x>\gamma_{2}-\frac{p-1}{p-2} v\right\}
$$

$w^{\prime}$ is given by

$$
w^{\prime}=1-\frac{N-1}{x} v-\frac{x}{\beta}+g(v) .
$$

Hence, because $g(v)<M$,

$$
w^{\prime}<1+M-\frac{1}{\beta}\left(\gamma_{2}-\frac{p-1}{p-2} B\right)=0
$$

in view of the definition of $\gamma_{2}$. Therefore, $F_{b}$ is part of the exit set of $S_{2}$.

On the back $F_{a}$ of $S_{2}$ :

$$
F_{a}=\left\{(v, w, x): 0<v<B, w>1, x=\gamma_{2}-\frac{p-1}{p-2} v\right\}
$$

an outward pointing normal vector $n_{a}$ is given by

$$
n_{a}=\left(-\frac{p-1}{p-2}, 0,-1\right) \text {. }
$$


Therefore, on $F_{a}$,

$$
n_{a} \cdot X=(p-1) v w^{p-2}(w-1)>0
$$

whence $F_{a}$ is also part of the exit set of $S_{2}$.

On the right-hand side $F_{r}$ of $S_{2}$ :

$$
F_{r}=\left\{(v, w, x): v=B, w \geq 1, x \geq \gamma_{2}-\frac{p-1}{p-2} B\right\}
$$

we have

$$
v^{\prime}=-(p-2) B w^{p-1}<0,
$$

so that $F_{r}$ is part of the entrance set of $S_{2}$.

Finally, as we saw in the proof of Lemma 5 , the left-hand side $F_{l}$ of $S_{2}$ is invariant.

Thus, we have found that the entrance set of $S_{2}$ is $F_{r}$. Therefore, as time is reversed, orbits can only leave $S_{2}$ through $F_{r}$.

In view of the definition of $\gamma_{2}, P_{\gamma} \in F_{l}$ if $\gamma>\gamma_{2}$. Since $e_{1}$ points into $S_{2}$, it follows that $U(t, \gamma) \in S_{2}$ if $\gamma>\gamma_{2}$ and $t$ is large. As we have seen $U(t, \gamma)$ can only leave $S$ through $F_{r}$. Because $F_{r} \cap F=\varnothing$ this means that $\gamma \notin G$ if $\gamma>\gamma_{2}$.

We may conclude from Lemmas 5 and 6 that as $t$ decreases from $+\infty, U\left(t, \gamma_{0}\right)$ enters $S$ and must leave it again through $F$ when $\gamma$ is small, and cannot leave it through $F$ when $\gamma$ is large. Thus, if $\gamma_{0}=\sup \{\gamma>0: \gamma \in G\}$ then $\gamma_{0}<\infty$. We shall show that $U\left(t, \gamma_{0}\right)$ is a solution of (IV) which passes through or tends to the half-line

$$
L_{0}=\{(v, w, x): v>0, w=0, x=0\}
$$

as $t$ decreases.

Let

$$
T_{0}=\inf \left\{T \in[-\infty, \infty): U\left(t, \gamma_{0}\right) \in S \text { for all } T<t<\infty\right\} .
$$

We shall consider the cases $T_{0}>-\infty$ and $T_{0}=-\infty$ in succession. (i) $T_{0}>-\infty$. Since $U(t, \gamma)$ depends continuously on $\gamma$ for $t$ bounded (Lemma 4), $U\left(T_{0}, \gamma_{0}\right) \in \partial F$. In addition, $x^{\prime}>0$ when $U \in S$, and therefore $U\left(T_{0}, \gamma_{0}\right) \in \partial F \cap\left\{x<\gamma_{0}\right\}$. However, the half-lines

$$
L_{1}=\{(v, w, x): v=0, w=0, x>0\}
$$

and

$$
L_{2}=\{(v, w, x): v=B, w=0, x>0\}
$$

are invariant. Therefore, we are left with the conclusion that $U\left(T_{0}, \gamma_{0}\right) \in L_{0} \cap$ $\{v \leq B\}$.

(ii) $T_{0}=-\infty$. In this case $x^{\prime}(t)>0$ and $x(t)>0$ for all $t \in \mathbf{R}$ and hence $x(t) \rightarrow x_{0}$ as $t \rightarrow-\infty$, where $x_{0} \geq 0$. Similarly, $v^{\prime}(t)<0$ and $v(t)<B$ for all $t \in \mathbf{R}$ whence $v(t) \rightarrow v_{0}$ as $t \rightarrow-\infty$, where $0<v_{0} \leq B$. It follows because $x^{\prime}$ has one sign that $\liminf _{t \rightarrow-\infty} x^{\prime}(t)=0$. This means, since $v_{0}>0$, that $\liminf _{t \rightarrow-\infty} w(t)=0$.

We assert that

(a) $x_{0}=0$;

(b) $\lim _{t \rightarrow-\infty} w(t)=0$.

To prove (a), suppose that $x_{0}>0$. Then there exists a sequence $\left\{t_{k}\right\}, t_{k} \rightarrow-\infty$ as $k \rightarrow \infty$ such that $U\left(t_{k}, \gamma_{0}\right) \rightarrow F \cup L_{2}$ as $k \rightarrow \infty$. However, because orbits leave $S$ through $F$ when time runs backward and $L_{2}$ is invariant, this possibility must be ruled out, and (a) is proved. 
Suppose now that (b) does not hold, i.e., there exists a number $\varepsilon>0$ and a sequence $\left\{\tau_{k}\right\}, \tau_{k} \rightarrow-\infty$ as $k \rightarrow \infty$ such that $w\left(\tau_{k}\right) \geq \varepsilon$ and $w^{\prime}\left(\tau_{k}\right)=0$ for all $k \geq 1$. Writing $v_{k}=v\left(\tau_{k}\right), w_{k}=w\left(\tau_{k}\right)$ and $x_{k}=x\left(\tau_{k}\right)$, the equation for $w^{\prime}$ becomes

$$
\frac{N-1}{x_{k}} v_{k} w_{k}^{p-1}=w_{k}^{p}-\frac{x_{k}}{\beta} w_{k}+g\left(v_{k}\right) \text {. }
$$

If we now let $k \rightarrow \infty$, the left-hand side becomes unbounded, whilst the right-hand side remains bounded. Hence, we obtain a contradiction, and (b) is proved as well.

We conclude from (a) and (b) that $U\left(t, \gamma_{0}\right) \rightarrow L_{0} \cap\{v \leq B\}$ as $t \rightarrow-\infty$.

Finally, we conclude that both if $T_{0}>-\infty$ and if $T_{0}=-\infty$, the orbit $\left\{U\left(t, \gamma_{0}\right)\right.$ : $\left.T_{0} \leq t<\infty\right\}$, where equality applies if $T_{0}>-\infty$, connects the half-line $L_{0}$ to the curve $C$.

This completes the proof of Theorem 1 .

\section{REFERENCES}

1. H. Brezis, L. A. Peletier and D. Terman, A very singular solution of the heat equation with absorption, Arch. Rational Mech. Anal. 95 (1986), 185-209.

2. M. Escobedo and O. Kavian, Variational problems related to self-similar solutions of the heat equation, Nonlinear Analysis (to appear).

3. A. Friedman and L. Veron, Solutions singulieres d'équations quasilinéaires elliptiques, C.R. Acad. Sci. Paris 302 (1986), 147-150.

4. _ Singular solutions of some quasilinear elliptic equations, Arch. Rational Mech. Anal. 96 (1986), 259-287.

5. J. Guckenheimer and P. J. Holmes, Nonlinear oscillations, dynamical systems and bifurcations of vector fields, Springer, New York, 1983.

6. S. Kamin and L. A. Peletier, Singular solutions of the heat equation with absorption, Proc. Amer. Math. Soc. 95 (1985), 205-210.

7. L. Oswald, Isolated, positive singularities for a non-linear heat equation, Houston J. Math. (to appear).

8. L. A. Peletier and D. Terman, A very singular solution of the porous media equation with absorption, J. Differential Equations 65 (1986), 396-410.

MAThematical Institute, University of Leiden, Leiden, The NetherLANDS

Department of Mathematics, Jilin University, ChangChun, Jilin, China 